\title{
New Biomarkers for Sepsis
}

\author{
Lixin Xie
}

Additional information is available at the end of the chapter

http://dx.doi.org/10.5772/50197

\section{Introduction}

Sepsis is the most important cause of morbidity and mortality in the intensive care unit (ICU), but it lacks specific clinical manifestations. As a result, sensitive and specific indicators of infection that can be collected easily and that accurately reflect infection severity and prognosis are highly coveted and are clinically important. Currently, common clinical indicators of infection include pyrexia, white blood cell (WBC) counts, C-reactive protein (CRP), and procalcitonin (PCT). However, in clinical settings, the limitation of CRP and PCT for assessing the severity and predicting prognosis may affect the clinician's ability to effectively evaluate the change in septic patients' general condition that would indicate deterioration and even impending death. Therefore, looking for new biomarkers with high sensitivity and specificity is one of the main research fields in sepsis. The objective of this paper is to review new biomarkers that are ....

\section{TREM-1}

Triggering receptor expressed on myeloid cells-1 (TREM-1) is a recently discovered member of the immunoglobulin superfamily of receptors that is expressed on polymorphonuclear granulocytes and mature monocytes. Bacterial or fungal infections may induce its expression.

\subsection{Soluble TREM-1}

sTREM-1 is a soluble form of TREM-1 that may be released into body fluids upon the upregulated expression of TREM-1(Bouchon A,et al. 2001). An increasing number of studies indicate that there are increased levels of sTREM-1 in body fluid samples for the following diseases and conditions: sepsis, pneumonia, pleural effusion, septic arthritis, meningitis, peritonitis, and uterine cavity infection (Gibot S, et al. 2004) (Gibot S, et al. 2004) (Liu CL, et al. 2007) (Collins CE,et al. 2009) (Determann RM, et al. 2006) (Kusanovic JP, et al.2010) 
(Determann RM, et al. 2009). This suggests that sTREM-1 may be a valuable diagnostic indicator for making distinctions between infectious and non-infectious diseases. It has also been found that septic shock patients have high levels of serum STREM-1 that are closely related to the severity of infection, and STREM-1 has a good positive correlation with the Sequential Organ Failure Assessment (SOFA) score (Gibot S, et al. 2004) (Dimopoulou I, et al. 2009). With regard to sepsis prognosis, dynamic changes in serum sTREM-1 may provide warnings concerning the life or death of patients (Zhang J, et al. 2011) (Gibot S, et al. 2005).

Urine sTREM-1 is more sensitive than WBC counts, serum CRP, and serum PCT for the early diagnosis of sepsis, as well as for dynamic assessments of severity and prognosis. It can also provide an early warning of possible secondary acute kidney injury (AKI) in sepsis patients (Su LX, et al. 2011).

In terms of diagnostic value for ventilator-associated pneumonia (VAP), the combination of sTREM-1 plus Clinical Pulmonary Infection Score (CPIS) improved the ability to diagnose VAP. Moreover, logistic regression analysis showed that sTREM-1 is an independent risk factor for VAP (Su LX, et al. for publication). We also found that sTREM-1 is of no use in determining bacteremia-caused, new fever in ICU patients, but sTREM-1 levels correlate with the prognosis of patients with bacteremia (Su LX, et al. for publication).

\subsection{Genetics and TREM-1}

More and more studies have confirmed that sepsis is caused by factors both environmental and genetic and that from the pathological point of view, genetic factors outweigh environmental factors. Therefore, clarification of how genetic factors are associated with sepsis may increase the awareness of susceptibility and prognosis concerning the disease. A study investigating an association between TREM-1 gene polymorphisms and severe sepsis concluded that 3 studied common polymorphisms within the TREM-1 gene (rs7768162, rs9471535, and rs2234237) may not play a major role in the predisposition to severe sepsis in a Chinese Han cohort (Chen Q, et al. 2008). However, Jung et al (Jung ES,et al. 2011) proved that TREM-1 SNPs (rs7768162, rs9471535, and rs2234237) may play a significant role in the development of intestinal Behcet's disease and may have modest effects on disease severity. Recently, in our study, we found that 2 variations (rs2234246 and rs2234237) within the TREM-1 gene are not correlated with susceptibility to sepsis. However, the TREM-1 rs2234237 polymorphism is associated with high 28-day mortality among sepsis patients, constituting a risk factor affecting prognosis (Su LX, et al. for publication). Therefore, TREM1 could be a fairly ideal genetic biomarker for the diagnosis and prognosis of sepsis.

\section{CD163}

CD163 is a transmembrane molecule, hitherto only discovered on the membrane of mononuclear phagocytes. As a specific scavenger receptor for hemoglobin/heme inside the body, it is capable of specific recognition of the hemoglobin-haptoglobin complex. Studies in recent years have found that CD163 regulates the expression of anti-inflammatory 
molecules, such as Interleukin-10 (IL-10) and Hemeoxigenase-1 (HO-1) (Moestrup SK, et al.2004) (Graversen JH, et al.2002).

\subsection{Soluble CD163}

Soluble CD163 (sCD163) comes from CD163 molecules that peel off the membrane of mononuclear cells (Moestrup SK, et al.2004) (Hogger P, et al. 2001). Blood levels of sCD163 have prognostic value for several inflammatory diseases and may have use in clinical applications as a biomarker of inflammatory diseases. Our prospective, clinical study confirmed that the serum sCD163 level might have potential value for the diagnosis of sepsis and severe sepsis, and its performance was superior to PCT and CRP levels. sCD163 also would have advantages for the dynamic monitoring of sepsis development and prognosis and have favorable prospects for use in clinical applications (Feng L, et al. for publication).

\subsection{Soluble CD163 and sepsis prognosis}

We compared sTREM-1, sCD163 and other clinical parameters for their assessment value for sepsis (Su LX, et al, for publication). On the day of ICU admission, the sepsis group displayed higher levels of serum sTREM-1, sCD163, PCT, and CRP than the Systemic Inflammatory Response Syndrome (SIRS) group $(P<.05)$. Although PCT, sTREM-1 and SOFA score were good markers to identify the severity of sepsis, sTREM-1 was the most reliable of these 3 markers. That is because serum sTREM-1 was a risk factor related to sepsis (OR=1.089, 95\% confidence interval [CI] 1.045-1.136, $P<.001)$. Its area under the Receiver Operating Characteristics (ROC) curve, meant for diagnosis, was 0.978 (95\% CI, 0.958-0.997), and that for severity evaluation was 0.9 (95\% CI, 0.823-0.977). Sensitivity and specificity were 0.91 and 0.87 respectively. On observation days 1, 3, 5, 7, 10, and 14, serum sCD163, sTREM-1, PCT and SOFA score continued to climb among non-survivors, while WBC and CRP levels decreased. In contrast, various indicators from the survivors showed a tendency to decline. The curves show that the non-survivors registered higher serum sTREM-1, sCD163, WBC and PCT levels, as well as SOFA score over an observation period of 14 days. Both sCD163 and SOFA score were independent factors impacting the survival time (sCD163 hazard ratio $=1.09,95 \% \mathrm{CI}, 1.035-1.154, P<.001$; SOFA score hazard ratio=1.23, 95\% CI, 1.126-1.335, $P<.001)$. Their areas under the ROC curve, denoting prognosis, measured 0.696 (95\% CI, 0.593-0.799) and 0.794 (95\% CI, 0.705-0.833), respectively. With 2.84 $\mathrm{mg} / \mathrm{L}$ as the cutoff point for sCD163, sensitivity measured 0.535 and specificity was 0.789 . In summary, the serum sCD163 level could be the most useful diagnostic value indicator for dynamic assessment of sepsis prognosis ( $\mathrm{Su}$ LX, et al, for publication) .

\subsection{Soluble CD163 and kidney disease}

Some studies in patients with bacteremia report high serum sCD163 expression, which has prognostic value (Gaini S, et al. 2008) (Moller HJ, et al. 2006), and high serum sCD163 expression also occurs in people with chronic kidney diseases (Axelsson J, et al. 2006). The 
CD163-hemoglobin scavenger receptor plays an important role in the process of the clearance and conversion of hemoglobin/heme in chronic kidney disease (Simoni J, et al. 2006). At present, it is unknown whether SCD163 can be detected in urine and what value it may possess for sepsis and secondary AKI. Recently, our team evaluated for the first time the potential value of urine sCD163 for sepsis and secondary AKI diagnosis, as well as for early assessment of prognosis. Our results demonstrated in an indirect manner the causes behind urine phagocyte increase and revealed a possible mechanism therein ( $\mathrm{Su} \mathrm{LX}$, et al. for publication). Perhaps this new discovery of a noninvasive detection index may have potential clinical value for sepsis-related multiple organ dysfunction.

\section{4. microRNAs}

MicroRNAs (miRNAs) are a type of endogenous non-coding small RNAs that are about 22 nucleotides in length (Lagos-Quintana et al. 2001) (Ambros 2004). They play important biological roles by inhibiting the expressions of messenger RNAs (mRNAs) (Krutzfeldt et al. 2006). As with mRNAs, some miRNAs are differentially expressed among tissues or developmental stages. Unlike some widely expressed miRNAs, these tissue- or developmental stage-specific miRNAs likely play key roles in regulating specific processes involved in the development or function of individual tissues (Etheridge et al. 2011). The liver-specific miR-122 has been applied in lipid and cholesterol metabolism, which are both known to be important functions of the liver (Bolmeson et al. 2011; Fernandez-Hernando et al. 2011). Because of their unique expression profiles, these miRNAs hold promise as diagnostic markers or therapeutic targets for many diseases. For example, miR-122 is required in hepatitis C virus (HCV) replication (Cermelli et al. 2011) and reagents that can modulate the level of miR-122 have moved into clinical development for HCV treatment (Pan et al. 2007; Said 2010; Zhang et al. 2010). miRNAs play an essential role in many physical and biological processes; thus, altered miRNA expression levels are associated with the occurrence and progression of disease.

\subsection{Circulating miRNAs}

A significant number of miRNAs have been observed outside of cells, within various body fluids. These cell-free miRNAs in body fluids are stable under harsh conditions including boiling, low or high $\mathrm{pH}$ and multiple freeze-thaw cycles (Chen et al. 2008; Mitchell et al. 2008). At present, there are 2 possible hypotheses for the stability and origin of circulating miRNAs. One hypothesis is that passive release occurs during tissue injury. For example, miRNA-216a was differentially expressed in the plasma of a pancreatic injury model in rat (Kong et al. 2010). miR-122 was also a biomarker for drug-induced liver injury (Wang et al. 2009). Alternatively, miRNAs are contained in small particles and are, therefore, protected against RNase activity. Recently, it has been shown that a transfer of mRNA and miRNA between cells can be accomplished through microvesicles (Valadi et al. 2007). These are small particles, which are derived from the cell plasma membrane into the extracellular space and released into the circulation (Caby et al. 2005; van Niel et al. 2006). Microvesicles 
are derived from various cell types, e.g. reticulocytes, dendritic cells, B and T cells and mast cells (Escola et al. 1998; Valenti et al. 2006; Brase et al. 2010). And in the peripheral blood, two-thirds of microvesicles are derived from platelets. Platelet-derived microvesicles play a role in angiogenesis and the metastatic spread of cancers (Janowska-Wieczorek et al. 2005). Platelet-derived microvesicles induce an immune response upon regulating gene expression in hematopoietic, endothelial, and monocytic cells (Setzer et al. 2006; Majka et al. 2007). Notably, platelet-derived microvesicle subpopulations are increased in patients with sepsis (Janiszewski et al. 2004). However it is currently unknown whether microvesicle content changes in these diseases (Hunter et al. 2008).

\subsection{What do we know about the miRNAs as biomarkers for sepsis?}

\subsection{1. miRNAs as prognostic biomarkers for sepsis}

Circulating miRNAs have been recently identified as biomarkers for sepsis. miR-150 was firstly identified as a prognostic marker for sepsis, and levels of miR-150, as detected by microarrays, were significantly different between the leukocytes of healthy controls and sepsis patients. In sepsis patients' plasma, levels of miR-150 were correlated with the level of SOFA score, and the plasma level ratio for miR-150/interleukin-18 can be used to evaluate sepsis severity (Vasilescu et al. 2009). A recent study demonstrated that miR150 differentially controls the development of natural killer (NK) and invariant NKT cell (iNKT) lineages by targeting the transcription factor c-Myb (Bezman et al. 2011). Few other functional studies about miR-150 in sepsis have been published. However, it has been demonstrated that the coding genes of tumor necrosis factor alpha (TNF-a), interleukin-10 (IL-10), and interleukin-18 (IL-18) have sequence complementarity to miR-150 (Vasilescu, Rossi et al. 2009). This finding suggests that miR-150 might be correlated with some of the immune system dysfunctions in sepsis patients, and it provides a new potential pathogenetic mechanism of sepsis. Hence, additional functional studies of miR-150 are required.

Sepsis is a complex disease that involves various tissues and organs. A simple screen for miRNAs differentially expressed in leukocytes may have missed many miRNAs secreted by other cell types. Hence, a genome-wide method was used to screen for differentially expressed miRNAs between the surviving and non-surviving groups of sepsis patients. Then, two novel prognostic biomarkers, miR-297 and miR-574-5p, were identified by microarray screening and quantitative real-time reverse transcription polymerase chain reaction (qRT-PCR) confirmation (Wang et al. 2012). miR-297 was more closely correlated with survival from sepsis, whereas miR-574-5p was correlated with death from sepsis. After analysis in a multivariable logistic regression model, results showed that a combination of sepsis stage, SOFA scores, and miR-574-5p were correlated with the death of sepsis patients. The predictive capability of these 3 combined variables was analyzed by a ROC curve; the area under the curve was 0.932 (95\% CI, 0.887-0.977). When the cutoff point was set at 0.288 , these 3 combined variables provided $78.13 \%$ sensitivity and $91.84 \%$ specificity. 
In summary, a genome-wide scan of sepsis patients' sera demonstrated that 2 miRNAs, miR-297 and miR-574-5p, might be related to the prognosis of sepsis in a genetic way. Identification of these miRNAs could provide more therapeutic targets for sepsis.

\subsection{2. miRNAs as diagnostic biomarkers for sepsis}

As diagnostic biomarkers for sepsis, levels of these markers should be not only differentially expressed between sepsis patients and healthy controls, but also between sepsis patients and SIRS patients. Levels of miR-146a and miR-223 in sepsis patients' sera were significantly decreased compared to SIRS patients and healthy controls. These levels were evaluated by qRT-PCR in 50 sepsis patients and 30 SIRS patients. The areas under the ROC curve for miR146a and miR-223 were 0.858 and 0.804 , respectively, which were both higher than IL-6 with an AUC of 0.785 (Wang et al. 2010). miR-146a regulates a pathway that promotes the binding of transcription repressor RelB to the TNF- $\alpha$ promoter, which generates facultative heterochromatin to silence acute proinflammatory genes (El Gazzar et al. 2011). This mechanism was proved in the THP-1 sepsis cell model of bacterial LPS/endotoxin tolerance. During LPS tolerance, transcriptional- and translation-repressive events combine to tightly regulate proinflammatory genes, which was a common feature of severe systemic inflammation (El Gazzar and McCall 2010). Hence, miR-146a was an important regulator during sepsis. Although the source and the release mechanism of miR-146a remain unknown, its clinical value is undeniable.

miR-15a and miR-16 are also newly identified diagnostic markers for sepsis. Levels of these 2 miRNAs in sepsis and SIRS patients were both significantly higher than in normal controls. And miR-15a can be used to distinguish sepsis patients from SIRS patients. The area under the ROC curve for miR-15a was 0.858 , which was much higher than the curves for CRP and PCT. These results were obtained from 166 sepsis patients and 32 SIRS patients (Wang et al, 2012). miR-15a and miR-16 were initially identified as tumor suppressors, and the dysregulation of these two miRNAs has been found to occur in many types of cancer (Calin et al. 2002; Bottoni et al. 2005; Bhattacharya et al. 2009; Yang et al. 2010; Bandi and Vassella 2011). Recently, decreases in miR-15a, miR-16 and miR-223 were found to be associated with the innate immune system by targeting I $\kappa$ B kinase alpha (IKK $\alpha$ ) mRNA, which is involved in the non-canonical NF- $\kappa B$ signaling pathway ( $\mathrm{Li}$ et al. 2010). I $\kappa$ B kinase (IKK) is an enzyme complex that is part of the upstream NF- $\kappa \mathrm{B}$ pathway. I $\kappa \mathrm{B} \alpha$ (inhibitor of kappa B) protein can inactivate NF- $\kappa \mathrm{B}$, and IKK can phosphorylate the inhibitory I $\kappa \mathrm{B} \alpha$ protein. Besides that, there is still no direct evidence for the correlations between miR-15a and miR-16 and sepsis. Hence, more functional studies of miR-15a and miR-16 need to be done.

For sepsis patients, timely diagnosis and early treatment are very important factors to improve their prognosis. miRNAs are newly identified as the main regulators of the immune system, and altered expression profiles in circulation can be used as diagnostic and prognostic biomarkers for sepsis. Although the functions of these miRNAs are not completely understood, their clinical value has been confirmed. New biomarkers also mean 
novel treatment targets. Hence, target genes of these miRNAs may emerge as potential treatment targets for sepsis patients.

\section{SNPs}

A single nucleotide polymorphism (SNP) is the most common type of stable genetic variation in the population. Thus, SNPs explain different sequence alternatives (alleles) existing at single base pair positions in genomic DNA in normal individuals in some populations. They are distinguished from rare variations by a requirement for the least abundant allele to have a frequency of $1 \%$ or more.(Brookes 1999)

A SNP occurs in approximately 1 of 1000 base pairs, with the most frequent being a $C$ to $T$ substitution. Polymorphisms, which occur both in the coding and non-coding genome regions, involve replacement of a nucleotide with another one, or insertion or deletion of 1 or more nucleotides. Because of a higher degree of preservation of exons to assure the functionality of genes, the frequency of polymorphisms in the non-coding regions is much higher compared with the coding ones. But changes in non-coding regions interfere with the structure and process of transcription and gene expression; thus, polymorphisms and mutations in non-coding regions may also produce a marked effect on phenotype presentations.(Prucha et al. 2008)

\subsection{Categories of SNPs}

SNPs are divided into two main categories, linked SNPs and causative SNPs. Linked SNPs (also called indicative SNPs) are located outside genes and do not affect protein function. Nevertheless, they are associated with a particular drug response or with the risk for getting a certain disease.

Causative SNPs affect the function of protein, correlating with a disease or influencing a person's response to medication. There are 2 forms of causative SNPs, coding SNPs (cSNPs) and non-coding SNPs. Coding SNPs, located in the coding region of a gene, can change the amino acid sequence of a gene's protein product; this type of SNP attracts more research than non-coding SNPs. Non-synonymous cSNPs (nsSNPs), which change the amino acid sequence of proteins and are likely to affect the structure and function of the proteins, are good candidates for disease-modifying alleles.(Jegga et al. 2007) And non-coding SNPs, located in the gene's regulatory sequences, also can change the level of gene expression. Because only about 3\% to $5 \%$ of a person's DNA sequence codes for the production of proteins, most SNPs are found outside of coding sequences.

\subsection{Influence of SNPs}

Single nucleotide substitutions may influence complex diseases by a variety of mechanisms. First, the amino acid sequence of some proteins whose functions include DNA binding, catalytic activity and receptor-ligand contact may be reduced or abolished by SNPs. Second, SNPs can interfere with the initiation or the termination codon or introduce errors in the 
reading frameshift. Third, mutations in known promoter motifs that alter DNA binding of transcription factors have the potential for decreasing or increasing gene expression. Finally, RNA cleavage-polyadenylation mutants in the untranslated region of the 5' UTR are thought to play a role in controlling mRNA translation while sequence variants in the $3^{\prime}$ UTR control RNA cleavage, stability, export and intracellular localization.(Wjst 2004) It is reported that only $10 \%$ of all gene-based SNPs have sequence-predicted functional relevance making them a primary target for genotyping in association studies. (Wjst 2004) There has been an effort to explain the potential causal relationship between the genetic changes and the development and course of diseases in order to modulate a patient's response to administration of drugs.(Sachidanandam et al. 2001)

\subsection{Researches in SNPs}

Genome-wide association (GWA) studies have been used to compare patient populations. The International HapMap Project and the arrival of technologies that type more than 100,000 SNPs in a single experiment have made genome-wide single nucleotide polymorphism (GW-SNP) assay a realistic endeavor. (Gibbs and Singleton 2006)

\subsection{SNPs as biomarkers for sepsis}

More than 20 years ago, Sorensen and colleagues reported that if one of an adult adoptee's biologic parents died of infection before the age of 50, the adoptee had a 5.81-fold increased risk of dying from infection.(Sorensen et al. 1988) Current sepsis-related polymorphism studies have most commonly focused on one or more polymorphisms for specific genes whose protein products are elements of biologic pathways implicated in sepsis. Many of these studies are association studies where various proinflammatory cytokines and their receptors, novel biomarkers, enzymes and mediators were compared with the development and clinical outcomes of sepsis, severe sepsis and organ dysfunction. In particular, identification of genetic variation in the Toll-like receptors (TLRs) and proinflammatory cytokines has provided valuable insights into the influence of genetic heterogeneity on the response to bacterial infection. And sometimes, different conclusions were given in researching the same SNP. Analyzing the variation in genes and associated differences in response to infection may contribute to the development of new gene diagnosis and therapeutic interventions that will improve outcome in this patient population.

\subsubsection{TLRS}

Expressed by macrophages, dendritic cells, neutrophils, and other cell populations, TLRs play a central role in the innate immune response to infection through the recognition of distinct bacterial antigens. (Leulier and Lemaitre 2008) TLR4 is crucial for the recognition of lipopolysaccharide (LPS), while TLR2 is essential in the recognition of Gram-positive bacterial components.(Martin 2000; Opal and Huber 2002) In an American research study, human subjects with 2 TLR mutations (299 Asp $\rightarrow$ Gly and $399 \mathrm{Thr} \rightarrow$ Ile) were compared to subjects with TLR4 wild type for response to inhaled toxins. The changes in 299 Asp $\rightarrow$ Gly, 
but not $399 \mathrm{Thr} \rightarrow$ Ile, significantly reduced nuclear levels of NF-kB in LPS-stimulated THP-1 cells. The 299/399 polymorphisms had reduced levels of IL-1a associated with hyporesponsiveness to inhaled endotoxin in humans. (Arbour et al. 2000) Patients with septic shock with the TLR4 Asp299Gly/Thr399Ile alleles had a higher prevalence of gramnegative infections.(Lorenz et al. 2002) Furthermore, the TLR4 299 polymorphism has been reported to be associated with severe sepsis, septic shock and a higher mortality in septic patients with SIRS. (Lorenz, Mira et al. 2002; Child et al. 2003; Barber et al. 2004) Some studies illustrate that TLR2 $753 \mathrm{Arg} \rightarrow \mathrm{Gln}$ and $677 \mathrm{Arg} \rightarrow \operatorname{Trp}$ may predispose individuals to certain gram-positive infections such as tuberculosis or leprosy.(Ben-Ali et al. 2004; Ogus et al. 2004)

\subsubsection{Cytokines}

A key role in the pathogenesis of sepsis is the balance or imbalance of pro- and antiinflammatory cytokines. Disorders of coagulation are common in sepsis, and $30 \%$ to $50 \%$ of patients have the more severe clinical form, disseminated intravascular coagulation.(Levi et al. 2000)

\section{TNF- $\alpha$}

TNF- $\alpha$, a pleiotropic cytokine mainly produced by activated monocytes and macrophages, plays a key role in the inflammatory response, and its overexpression can lead to the progression of inflammatory and autoimmune diseases.(Locksley et al. 2001; O'Shea et al. 2002) But the association between TNF gene polymorphisms and morbidity or clinical outcome of sepsis was not so clearly defined. An association between development of sepsis, but not mortality from sepsis, and the TNF2 genotype in the overall population was found.(Teuffel et al. 2010) An Austrian study discovered that peak values of inflammatory and coagulation markers were not different between wild-type TNF- $\alpha-308$ individuals (GG) and carriers of the TNF- $\alpha-308$ mutant allele (GA and AA).(Kovar et al. 2007)

\section{IL-1 and receptor}

The interleukin-1 (IL-1) receptor-associated kinase 1 (IRAK1) is believed to play an important role in TLR2- and TLR4-induced activation of NF-KB, a critical event in the transcriptional regulation of many sepsis-associated proinflammatory mediators.(Arcaroli et al. 2006) Alleles A2, B2 and RN2 in the IL-1 gene might be important high-risk genetic markers for sepsis.(Ma et al. 2002) IRAK1 might be a genetic risk factor for the occurrence and development of sepsis in the Chinese population. (Arcaroli, Silva et al. 2006)

\section{$I L-10$}

Interleukin-10 (IL-10) is an anti-inflammatory cytokine produced by macrophages and Thelper-type II (TH2) lymphocytes that can downregulate inflammatory production, which plays a very important role in the process of induction of immunoparalysis. (Nicod et al. 
1995; Thomassen et al. 1996) Three SNPs $(-1082,-819$, and -592) were found in the regulatory region of the IL-10 gene. The A allele of the -1082 polymorphism in the IL-10 gene promoter is associated with late blood stream infections in ventilated, very low-birthweight infants and with sepsis susceptibility, whereas the $G$ allele is associated with higher stimulated IL-10 production and increased mortality in severe sepsis.(Shu et al. 2003; Stanilova et al. 2006) Otherwise, the $-1082 \mathrm{G} / \mathrm{G}$ genotype has been associated with lower mortality and organ failure among the subjects with acute respiratory distress syndrome.(Gong et al. 2006) The A allele of the single nucleotide polymorphism at -592 base pairs was associated with higher mortality in sepsis.(Lowe et al. 2003)

Sepsis, an increasing cause of mortality in patients with infectious diseases, especially in seriously ill patients in the ICU, requires rapid diagnosis and treatment. Because SNPs occur frequently throughout the genome and tend to be relatively stable genetically, they can be used as excellent biological markers in sepsis. Depending on rapid advances in technology and informatics, the primary goal in the management of sepsis may change from rapid treatment to prevention for those most at risk. The health care cost savings from such changes could be substantial.

\section{Conclusion}

In conclusion, the search for new biomarkers for assessing the severity of sepsis patients and predicting prognosis is very important, interesting, and challenging work, providing new insights to confront sepsis.

\section{Author details}

Lixin Xie

Department of Respiratory Medicine, Hainan Branch of Chinese PLA General Hospital, China

Vice Director of Department of Respiratory Medicine, Chinese PLA General Hospital, China

\section{References}

Bouchon A, Facchetti F, Weigand MA, Colonna M, (2001) TREM-1 amplifies inflammation and is a crucial mediator of septic shock. Nature 410: 1103-1107

Gibot S, Kolopp-Sarda MN, Bene MC, Cravoisy A, Levy B, Faure GC, Bollaert PE, (2004) Plasma level of a triggering receptor expressed on myeloid cells-1: its diagnostic accuracy in patients with suspected sepsis. Ann Intern Med 141: 9-15

Gibot S, Cravoisy A, Levy B, Bene MC, Faure G, Bollaert PE, (2004) Soluble triggering receptor expressed on myeloid cells and the diagnosis of pneumonia. N Engl J Med 350: 451-458

Liu CL, Hsieh WY, Wu CL, Kuo HT, Lu YT, (2007) Triggering receptor expressed on myeloid cells-1 in pleural effusions: a marker of inflammatory disease. Respir Med 101: 903-909 
Collins CE, La DT, Yang HT, Massin F, Gibot S, Faure G, Stohl W, (2009) Elevated synovial expression of triggering receptor expressed on myeloid cells 1 in patients with septic arthritis or rheumatoid arthritis. Ann Rheum Dis 68: 1768-1774

Determann RM, Weisfelt M, de Gans J, van der Ende A, Schultz MJ, van de Beek D, (2006) Soluble triggering receptor expressed on myeloid cells 1: a biomarker for bacterial meningitis. Intensive Care Med 32: 1243-1247

Kusanovic JP, Romero R, Chaiworapongsa T, Mittal P, Mazaki-Tovi S, Vaisbuch E, Erez O, Gotsch F, Than NG, Edwin SS, Pacora P, Jodicke C, Yeo L, Hassan SS, (2010) Amniotic fluid STREM-1 in normal pregnancy, spontaneous parturition at term and preterm, and intra-amniotic infection/inflammation. J Matern Fetal Neonatal Med 23: 34-47

Determann RM, van Till JW, van Ruler O, van Veen SQ, Schultz MJ, Boermeester MA, (2009) sTREM-1 is a potential useful biomarker for exclusion of ongoing infection in patients with secondary peritonitis. Cytokine 46: 36-42

Gibot S, Kolopp-Sarda MN, Bene MC, Bollaert PE, Lozniewski A, Mory F, Levy B, Faure GC, (2004) A soluble form of the triggering receptor expressed on myeloid cells-1 modulates the inflammatory response in murine sepsis. J Exp Med 200: 1419-1426

Dimopoulou I, Orfanos SE, Pelekanou A, Kotanidou A, Livaditi O, Augustatou C, Zervou M, Douka E, Theodorakopoulou M, Karagianni V, Douzinas E, Armaganidis A, Giamarellos-Bourboulis EJ, (2009) Serum of patients with septic shock stimulates the expression of Trem-1 on U937 monocytes. Inflamm Res 58: 127-132

Zhang J, She D, Feng D, Jia Y, Xie L, (2011) Dynamic changes of serum soluble triggering receptor expressed on myeloid cells-1 (sTREM-1) reflect sepsis severity and can predict prognosis: a prospective study. BMC Infect Dis 11: 53

Gibot S, Cravoisy A, Kolopp-Sarda MN, Bene MC, Faure G, Bollaert PE, Levy B, (2005) Timecourse of sTREM (soluble triggering receptor expressed on myeloid cells)-1, procalcitonin, and C-reactive protein plasma concentrations during sepsis. Crit Care Med 33: 792-796

Su LX, Feng L, Zhang J, Xiao YJ, Jia YH, Yan P, Feng D, Xie LX, (2011) Diagnostic value of urine sTREM-1 for sepsis and relevant acute kidney injuries: a prospective study. Crit Care 15: R250

Long-xiang Su KM, Xin Zhang, Hui-juan Wang, Peng Yan, Yan-hong Jia, Dan Feng, Li-xin Xie, Diagnostic Value of sTREM-1 and Procalcitonin Serum Levels and Pulmonary Infection Score for Ventilator-associated Pneumonia among ICU Sepsis Patients

Long-xiang Su B-cH, Li-ling Liang, Zhao-xu Jiang, Jie Deng, Yan-hong Jia, Dan Feng, Li-xin Xie, Value of Soluble TREM-1, Procalcitonin, and C-reactive Protein Serum Levels as Markers for Detecting Bacteremia among Sepsis Patients with New Fever in Intensive Care Units

Chen Q, Zhou H, Wu S, Wang H, Lv C, Cheng B, Xie G, Fang X, (2008) Lack of association between TREM-1 gene polymorphisms and severe sepsis in a Chinese Han population. Human immunology 69: 220-226

Jung ES, Kim SW, Moon CM, Shin DJ, Son NH, Kim ES, Lee HJ, Hong SP, Kim TI, Kim WH, Cheon JH, (2011) Relationships between genetic polymorphisms of triggering receptor expressed on myeloid cells-1 and inflammatory bowel diseases in the Korean population. Life sciences 89: 289-294 
Long-xiang Su Z-xJ, Xin Zhang, Dan Feng, Li-xin Xie, Dynamic changes of serum soluble triggering receptor expressed on myeloid cells-1 (sTREM-1) and its gene polymorphisms are associated with sepsis prognosis

Lin Feng XZ, Long-Xiang Su,Dan Feng,Yan-Hong Jia,Li-Xin Xie, Clinical Significance of Soluble Hemoglobin Scavenger Receptor CD163 (sCD163) inSepsis, a Prospective Study

Long-xiang Su LF, Qing Song, Hong-jun Kang, Xin-gang Zhang, Zhi-xin Liang, Yan-hong Jia, Dan Feng, Li-xin Xie, Determining the Best Diagnostic Biomarker for Sepsis and Prognosis Assessment

Moestrup SK, Moller HJ, (2004) CD163: a regulated hemoglobin scavenger receptor with a role in the anti-inflammatory response. Annals of medicine 36: 347-354

Graversen JH, Madsen M, Moestrup SK, (2002) CD163: a signal receptor scavenging haptoglobin-hemoglobin complexes from plasma. The international journal of biochemistry \& cell biology 34: 309-314

Hogger P, Sorg C, (2001) Soluble CD163 inhibits phorbol ester-induced lymphocyte proliferation. Biochemical and biophysical research communications 288: 841-843

Gaini S, Pedersen SS, Koldkaer OG, Pedersen C, Moestrup SK, Moller HJ, (2008) New immunological serum markers in bacteraemia: anti-inflammatory soluble CD163, but not proinflammatory high mobility group-box 1 protein, is related to prognosis. Clinical and experimental immunology 151: 423-431

Moller HJ, Moestrup SK, Weis N, Wejse C, Nielsen H, Pedersen SS, Attermann J, Nexo E, Kronborg G, (2006) Macrophage serum markers in pneumococcal bacteremia: Prediction of survival by soluble CD163. Critical care medicine 34: 2561-2566

Axelsson J, Moller HJ, Witasp A, Qureshi AR, Carrero JJ, Heimburger O, Barany P, Alvestrand A, Lindholm B, Moestrup SK, Stenvinkel P, (2006) Changes in fat mass correlate with changes in soluble sCD163, a marker of mature macrophages, in patients with CKD. American journal of kidney diseases : the official journal of the National Kidney Foundation 48: 916-925

Simoni J, Simoni G, Griswold JA, Moeller JF, Tsikouris JP, Khanna A, Roongsritong C, Wesson DE, (2006) Role of free hemoglobin in 8-iso prostaglandin F2-alpha synthesis in chronic renal failure and its impact on CD163-Hb scavenger receptor and on coronary artery endothelium. ASAIO J 52: 652-661

Long-xiang Su LF, Zhao-xu Jiang, Ming Li, Kun Xiao, Peng Yan, Dan Feng, Li-xin Xie, Diagnostic value of urine sCD163 for sepsis and relevant acute kidney injuries: a prospective study

Ambros, V. (2004).The functions of animal microRNAs. Nature VOL.431, NO.7006, (2004): PP. 350-355, ISSN 1476-4687.

Bandi, N. and E. Vassella (2011).miR-34a and miR-15a/16 are co-regulated in non-small cell lung cancer and control cell cycle progression in a synergistic and $\mathrm{Rb}$-dependent manner. Mol Cancer VOL.10, (2011): PP. 55, ISSN 1476-4598.

Bezman, N. A., T. Chakraborty, et al. (2011).miR-150 regulates the development of NK and iNKT cells. J Exp Med VOL.208, NO.13, (2011): PP. 2717-2731, ISSN 1540-9538.

Bhattacharya, R., M. Nicoloso, et al. (2009).MiR-15a and MiR-16 control Bmi-1 expression in ovarian cancer. Cancer Res VOL.69, NO.23, (2009): PP. 9090-9095, ISSN 1538-7445. 
Bolmeson, C., J. L. Esguerra, et al. (2011).Differences in islet-enriched miRNAs in healthy and glucose intolerant human subjects. Biochem Biophys Res Commun VOL.404, NO.1, (2011): PP. 16-22, ISSN 1090-2104.

Bottoni, A., D. Piccin, et al. (2005).miR-15a and miR-16-1 down-regulation in pituitary adenomas. J Cell Physiol VOL.204, NO.1, (2005): PP. 280-285, ISSN 0021-9541.

Brase, J. C., D. Wuttig, et al. (2010).Serum microRNAs as non-invasive biomarkers for cancer. Mol Cancer VOL.9, (2010): PP. 306, ISSN 1476-4598.

Caby, M. P., D. Lankar, et al. (2005).Exosomal-like vesicles are present in human blood plasma. Int Immunol VOL.17, NO.7, (2005): PP. 879-887, ISSN 0953-8178.

Wang H. J., Meng, et al. (2012). Evidence for serum miR-15a and miR-16 levels as bimarkers that distinguish sepsis from SIRS in human subjects. Clin Chem and Lab Med. VOL.50, NO.8, (2012).

Calin, G. A., C. D. Dumitru, et al. (2002).Frequent deletions and down-regulation of microRNA genes miR15 and miR16 at 13q14 in chronic lymphocytic leukemia. Proc Natl Acad Sci U S A VOL.99, NO.24, (2002): PP. 15524-15529, ISSN 0027-8424.

Cermelli, S., A. Ruggieri, et al. (2011).Circulating microRNAs in patients with chronic hepatitis C and non-alcoholic fatty liver disease. PLoS One VOL.6, NO.8, (2011): PP. e23937, ISSN 1932-6203.

Chen, X., Y. Ba, et al. (2008).Characterization of microRNAs in serum: a novel class of biomarkers for diagnosis of cancer and other diseases. Cell Res VOL.18, NO.10, (2008): PP. 997-1006, ISSN 1748-7838.

El Gazzar, M., A. Church, et al. (2011).MicroRNA-146a regulates both transcription silencing and translation disruption of TNF-alpha during TLR4-induced gene reprogramming. $J$ Leukoc Biol VOL.90, NO.3, (2011): PP. 509-519, ISSN 1938-3673.

El Gazzar, M. and C. E. McCall (2010).MicroRNAs distinguish translational from transcriptional silencing during endotoxin tolerance. J Biol Chem VOL.285, NO.27, (2010): PP. 20940-20951, ISSN 1083-351X.

Escola, J. M., M. J. Kleijmeer, et al. (1998).Selective enrichment of tetraspan proteins on the internal vesicles of multivesicular endosomes and on exosomes secreted by human Blymphocytes. J Biol Chem VOL.273, NO.32, (1998): PP. 20121-20127, ISSN 0021-9258.

Etheridge, A., I. Lee, et al. (2011).Extracellular microRNA: a new source of biomarkers. Mutat Res VOL.717, NO.1-2, (2011): PP. 85-90, ISSN 0027-5107.

Fernandez-Hernando, C., Y. Suarez, et al. (2011).MicroRNAs in lipid metabolism. Curr Opin Lipidol VOL.22, NO.2, (2011): PP. 86-92, ISSN 1473-6535.

Hunter, M. P., N. Ismail, et al. (2008).Detection of microRNA expression in human peripheral blood microvesicles. PLoS One VOL.3, NO.11, (2008): PP. e3694, ISSN 1932-6203.

Janiszewski, M., A. O. Do Carmo, et al. (2004).Platelet-derived exosomes of septic individuals possess proapoptotic $\mathrm{NAD}(\mathrm{P}) \mathrm{H}$ oxidase activity: A novel vascular redox pathway. Crit Care Med VOL.32, NO.3, (2004): PP. 818-825, ISSN 0090-3493.

Janowska-Wieczorek, A., M. Wysoczynski, et al. (2005).Microvesicles derived from activated platelets induce metastasis and angiogenesis in lung cancer. Int J Cancer VOL.113, NO.5, (2005): PP. 752-760, ISSN 0020-7136. 
Kong, X. Y., Y. Q. Du, et al. (2010).Plasma miR-216a as a potential marker of pancreatic injury in a rat model of acute pancreatitis. World J Gastroenterol VOL.16, NO.36, (2010): PP. 4599-4604, ISSN 1007-9327.

Krutzfeldt, J., M. N. Poy, et al. (2006).Strategies to determine the biological function of microRNAs. Nat Genet VOL.38 Suppl, (2006): PP. S14-19, ISSN 1061-4036.

Lagos-Quintana, M., R. Rauhut, et al. (2001).Identification of novel genes coding for small expressed RNAs. Science VOL.294, NO.5543, (2001): PP. 853-858, ISSN 0036-8075.

Li, T., M. J. Morgan, et al. (2010).MicroRNAs modulate the noncanonical transcription factor NF-kappaB pathway by regulating expression of the kinase IKKalpha during macrophage differentiation. Nat Immunol VOL.11, NO.9, (2010): PP. 799-805, ISSN 1529-2916.

Majka, M., J. Kijowski, et al. (2007).Evidence that platelet-derived microvesicles may transfer platelet-specific immunoreactive antigens to the surface of endothelial cells and CD34+ hematopoietic stem/ progenitor cells--implication for the pathogenesis of immune thrombocytopenias. Folia Histochem Cytobiol VOL.45, NO.1, (2007): PP. 27-32, ISSN 02398508.

Mitchell, P. S., R. K. Parkin, et al. (2008).Circulating microRNAs as stable blood-based markers for cancer detection. Proc Natl Acad Sci U S A VOL.105, NO.30, (2008): PP. 10513-10518, ISSN 1091-6490.

Pan, Q. W., S. D. Henry, et al. (2007).New therapeutic opportunities for hepatitis C based on small RNA. World J Gastroenterol VOL.13, NO.33, (2007): PP. 4431-4436, ISSN 1007-9327.

Said, E. A. (2010).The Need for New Anti-Hepatitis C Virus Therapeutic Strategies: Targeting the cellular micro-ribonucleic acids? Sultan Qaboos Univ Med J VOL.10, NO.3, (2010): PP. 312-317, ISSN 2075-0528.

Setzer, F., V. Oberle, et al. (2006).Platelet-derived microvesicles induce differential gene expression in monocytic cells: a DNA microarray study. Platelets VOL.17, NO.8, (2006): PP. 571-576, ISSN 0953-7104.

Valadi, H., K. Ekstrom, et al. (2007).Exosome-mediated transfer of mRNAs and microRNAs is a novel mechanism of genetic exchange between cells. Nat Cell Biol VOL.9, NO.6, (2007): PP. 654-659, ISSN 1465-7392.

Valenti, R., V. Huber, et al. (2006).Human tumor-released microvesicles promote the differentiation of myeloid cells with transforming growth factor-beta-mediated suppressive activity on T lymphocytes. Cancer Res VOL.66, NO.18, (2006): PP. 92909298, ISSN 0008-5472.

van Niel, G., I. Porto-Carreiro, et al. (2006).Exosomes: a common pathway for a specialized function. J Biochem VOL.140, NO.1, (2006): PP. 13-21, ISSN 0021-924X.

Vasilescu, C., S. Rossi, et al. (2009).MicroRNA fingerprints identify miR-150 as a plasma prognostic marker in patients with sepsis. PLoS One VOL.4, NO.10, (2009): PP. e7405, ISSN 1932-6203.

Wang, H., K. Meng, et al. (2012).Serum miR-574-5p: A Prognostic Predictor of Sepsis Patients. Shock VOL.37, NO.3, (2012): PP. 263-267, ISSN 1540-0514.

Wang, J. F., M. L. Yu, et al. (2010).Serum miR-146a and miR-223 as potential new biomarkers for sepsis. Biochem Biophys Res Commun VOL.394, NO.1, (2010): PP. 184-188, ISSN 1090-2104. 
Wang, K., S. Zhang, et al. (2009).Circulating microRNAs, potential biomarkers for druginduced liver injury. Proc Natl Acad Sci U S A VOL.106, NO.11, (2009): PP. 4402-4407, ISSN 1091-6490.

Yang, Y., X. Li, et al. (2010).The role of microRNA in human lung squamous cell carcinoma. Cancer Genet Cytogenet VOL.200, NO.2, (2010): PP. 127-133, ISSN 1873-4456.

Zhang, G., Q. Wang, et al. (2010).Therapeutics Based on microRNA: A New Approach for Liver Cancer. Curr Genomics VOL.11, NO.5, (2010): PP. 311-325, ISSN 1875-5488.

Arbour, N. C., E. Lorenz, et al. (2000).TLR4 mutations are associated with endotoxin hyporesponsiveness in humans. Nat Genet VOL.25, NO.2, (2000): PP. 187-191, ISSN 1061-4036.

Arcaroli, J., E. Silva, et al. (2006).Variant IRAK-1 haplotype is associated with increased nuclear factor-kappa B activation and worse outcomes in sepsis. American Journal of Respiratory and Critical Care Medicine VOL.173, NO.12, (2006): PP. 1335-1341, ISSN 1073-449X.

Barber, R. C., C. C. Aragaki, et al. (2004).TLR4 and TNF-alpha polymorphisms are associated with an increased risk for severe sepsis following burn injury. J Med Genet VOL.41, NO.11, (2004): PP. 808-813, ISSN 1468-6244.

Ben-Ali, M., M. R. Barbouche, et al. (2004).Toll-like receptor 2 Arg677Trp polymorphism is associated with susceptibility to tuberculosis in Tunisian patients. Clin Diagn Lab Immunol VOL.11, NO.3, (2004): PP. 625-626, ISSN 1071-412X.

Brookes, A. J. (1999).The essence of SNPs. Gene VOL.234, NO.2, (1999): PP. 177-186, ISSN 0378-1119.

Child, N. J., I. A. Yang, et al. (2003).Polymorphisms in Toll-like receptor 4 and the systemic inflammatory response syndrome. Biochem Soc Trans VOL.31, NO.Pt 3, (2003): PP. 652653, ISSN 0300-5127.

Gibbs, J. R. and A. Singleton (2006).Application of genome-wide single nucleotide polymorphism typing: simple association and beyond. PLoS Genet VOL.2, NO.10, (2006): PP. e150, ISSN 1553-7404.

Gong, M. N., B. T. Thompson, et al. (2006).Interleukin-10 polymorphism in position -1082 and acute respiratory distress syndrome. Eur Respir J VOL.27, NO.4, (2006): PP. 674-681, ISSN 0903-1936.

Jegga, A. G., S. Gowrisankar, et al. (2007).PolyDoms: a whole genome database for the identification of non-synonymous coding SNPs with the potential to impact disease. Nucleic Acids Res VOL.35, NO.Database issue, (2007): PP. D700-706, ISSN 1362-4962.

Kovar, F. M., C. Marsik, et al. (2007).The tumor necrosis factor alpha -308 G/A polymorphism does not influence inflammation and coagulation response in human endotoxemia. Shock VOL.27, NO.3, (2007): PP. 238-241, ISSN 1073-2322.

Leulier, F. and B. Lemaitre (2008).Toll-like receptors--taking an evolutionary approach. Nat Rev Genet VOL.9, NO.3, (2008): PP. 165-178, ISSN 1471-0064.

Levi, M., E. de Jonge, et al. (2000).[Disseminated intravascular coagulation]. Ned Tijdschr Geneeskd VOL.144, NO.10, (2000): PP. 470-475, ISSN 0028-2162.

Locksley, R. M., N. Killeen, et al. (2001).The TNF and TNF receptor superfamilies: integrating mammalian biology. Cell VOL.104, NO.4, (2001): PP. 487-501, ISSN 0092-8674. 
Lorenz, E., J. P. Mira, et al. (2002).Relevance of mutations in the TLR4 receptor in patients with gram-negative septic shock. Arch Intern Med VOL.162, NO.9, (2002): PP. 1028-1032, ISSN 0003-9926.

Lowe, P. R., H. F. Galley, et al. (2003).Influence of interleukin-10 polymorphisms on interleukin-10 expression and survival in critically ill patients. Crit Care Med VOL.31, NO.1, (2003): PP. 34-38, ISSN 0090-3493.

Ma, P., D. Chen, et al. (2002).[Genomic polymorphism within interleukin-1 family cytokines influences the outcome of septic patients]. Zhonghua Yi Xue Za Zhi VOL.82, NO.18, (2002): PP. 1237-1241, ISSN 0376-2491.

Martin, T. R. (2000).Recognition of bacterial endotoxin in the lungs. Am J Respir Cell Mol Biol VOL.23, NO.2, (2000): PP. 128-132, ISSN 1044-1549.

Nicod, L. P., F. el Habre, et al. (1995).Interleukin-10 decreases tumor necrosis factor alpha and beta in alloreactions induced by human lung dendritic cells and macrophages. Am J Respir Cell Mol Biol VOL.13, NO.1, (1995): PP. 83-90, ISSN 1044-1549.

O'Shea, J. J., A. Ma, et al. (2002).Cytokines and autoimmunity. Nat Rev Immunol VOL.2, NO.1, (2002): PP. 37-45, ISSN 1474-1733.

Ogus, A. C., B. Yoldas, et al. (2004).The Arg753GLn polymorphism of the human toll-like receptor 2 gene in tuberculosis disease. Eur Respir J VOL.23, NO.2, (2004): PP. 219-223, ISSN 0903-1936.

Opal, S. M. and C. E. Huber (2002).Bench-to-bedside review: Toll-like receptors and their role in septic shock. Crit Care VOL.6, NO.2, (2002): PP. 125-136, ISSN 1364-8535.

Prucha, M., R. Zazula, et al. (2008).Genomic polymorphism and sepsis--is there a reason for optimism? Prague Med Rep VOL.109, NO.2-3, (2008): PP. 113-126, ISSN 1214-6994.

Sachidanandam, R., D. Weissman, et al. (2001).A map of human genome sequence variation containing 1.42 million single nucleotide polymorphisms. Nature VOL.409, NO.6822, (2001): PP. 928-933, ISSN 0028-0836.

Shu, Q., X. Fang, et al. (2003).IL-10 polymorphism is associated with increased incidence of severe sepsis. Chin Med J (Engl) VOL.116, NO.11, (2003): PP. 1756-1759, ISSN 0366-6999.

Sorensen, T. I., G. G. Nielsen, et al. (1988).Genetic and environmental influences on premature death in adult adoptees. $N$ Engl J Med VOL.318, NO.12, (1988): PP. 727-732, ISSN 0028-4793.

Stanilova, S. A., L. D. Miteva, et al. (2006).Interleukin-10-1082 promoter polymorphism in association with cytokine production and sepsis susceptibility. Intensive Care Med VOL.32, NO.2, (2006): PP. 260-266, ISSN 0342-4642.

Teuffel, O., M. C. Ethier, et al. (2010).Association between tumor necrosis factor-alpha promoter $-308 \mathrm{~A} / \mathrm{G}$ polymorphism and susceptibility to sepsis and sepsis mortality: a systematic review and meta-analysis. Crit Care Med VOL.38, NO.1, (2010): PP. 276-282, ISSN 1530-0293.

Thomassen, M. J., L. T. Divis, et al. (1996).Regulation of human alveolar macrophage inflammatory cytokine production by interleukin-10. Clin Immunol Immunopathol VOL.80, NO.3 Pt 1, (1996): PP. 321-324, ISSN 0090-1229.

Wjst, M. (2004).Target SNP selection in complex disease association studies. BMC Bioinformatics VOL.5, (2004): PP. 92, ISSN 1471-2105. 